\title{
Effects of a pro-oxidant dietary supplement on cell-mediated immunity in broilers
}

\author{
I. C. Scurtu ${ }^{1}$, G. Giurgiu ${ }^{1}$, M. Mircean ${ }^{1}$, M. Spinu ${ }^{2}$, C. Sandru ${ }^{2}$, M. Niculae ${ }^{2}$ and L. Livitchi ${ }^{1}$ \\ ${ }^{1}$ Internal Medicine Department and ${ }^{2}$ Infectious Diseases Department, Faculty of Veterinary Medicine, University of \\ Agricultural Sciences and Veterinary Medicine Cluj-Napoca, Manastur Street No. 3-5, 400372, Romania
}

Dietary components can impact on the immune response effectiveness by several mechanisms. Nevertheless, different authors questioned the opportunity of the antioxidant supplementation as a protective major against free radical (FR) production and suggested that this may have a negative influence on the immunological functions, while others pointed out the protecting effect of antioxidants on lymphocyte cells against oxidative stress ${ }^{(1,2)}$. The aim of this study was to investigate the effects of a pro-oxidant on the in vitro immune reactivity of broilers. The research was carried out on a total of 40 broilers divided into two experimental groups (A and B). In group B, sunflower oil oxidised by maintaining it at $110^{\circ} \mathrm{C}$ for $48 \mathrm{~h}$ as was supplemented in the basal diet as a pro-oxidative factor. The lipid oxidation level in sunflower oil, breast and thigh muscles and liver of broilers was established by measuring thiobarbituric acid reactive substances (TBARS). The impact on cell-mediated immunity was evaluated by determining the blood lymphocyte proliferation and the phagocyte activity. The blast transformation test assessed the lymphocytes proliferation intensity (stimulation indices, \%) were calculated after a $48 \mathrm{~h}$ contact with standard mitogens such as phytohaemagglutinin (PHA), lipopolysaccharide (LPS) and Concanavalin A (Con A) and also with sage (Salvia officinalis) alcoholic extracts well known for its antioxidant potential ${ }^{(3)}$. Also, the in vitro carbon clearance assay was performed to observe the phagocytes activity, expressed as the negative of the optical density slope over time (ln), during three time moments $(0,15$ and 30); for each experimental group, 4 variants were tested (control and alcohol, Rosmarinus officinalis and S. officinalis treated).

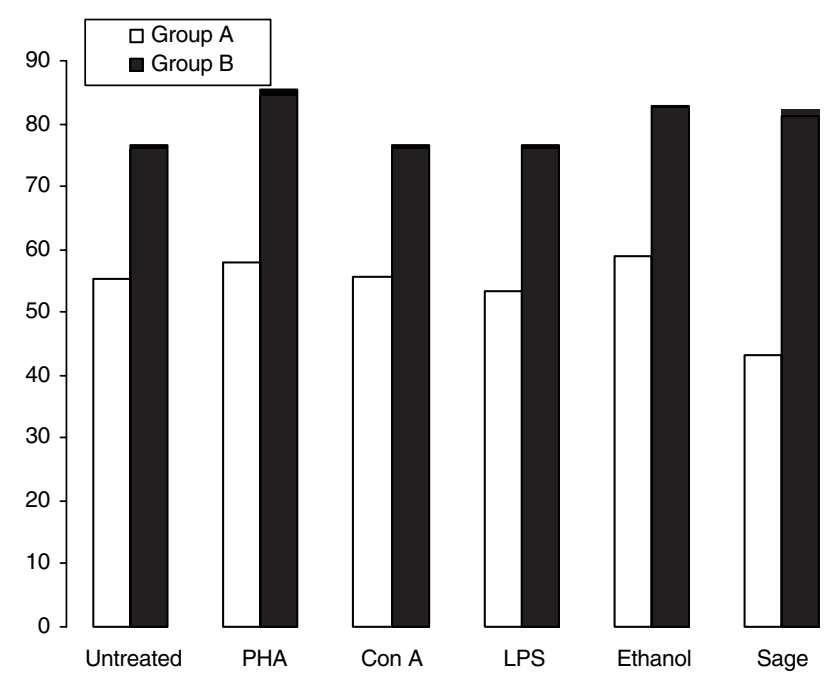

Fig. 1. Lymphocytes proliferation intensity (means of stimulation indices).

The results of blast transformation test indicated higher lymphocytes reactivity for group B when compared to group A (Fig. 1). Similar aspects were observed in the phagocyte activity, although this increase cannot be considered significant $(P>0.05)$.

These data suggest that the pro-oxidant supplementation along with the increasing effect on lipid peroxidation, augmented the lymphocytes and phagocytes reactivity, modulating both specific and non-specific cell-mediated immunity in broilers.

1. Knight JA (2000) Ann Clin Lab Sci 30, 145-158.

2. Hesabi Nameghi A, Nassiri Moghaddam H, Tavakkol Afshari J et al. (2007) J Anim Vet Adv 6, 1060-1069.

3. Lu YR \& Yeap Foo L (2001) Food Chem 75, 197-202. 